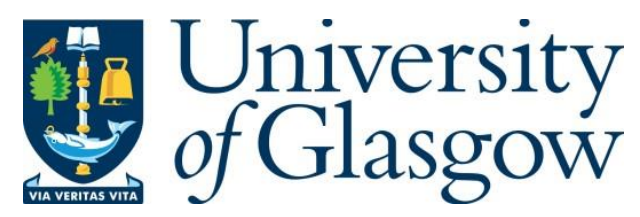

Eisele, F., Geline, M., Kessar, R. and Linckelmann, M. (2018) On Tate duality and a projective scalar property for symmetric algebras. Pacific Journal of Mathematics, 293(2), pp. 277-300.

There may be differences between this version and the published version. You are advised to consult the publisher's version if you wish to cite from it.

http://eprints.gla.ac.uk/177221/

Deposited on: 8 January 2019

Enlighten - Research publications by members of the University of Glasgow http://eprints.gla.ac.uk 


\title{
ON TATE DUALITY AND A PROJECTIVE SCALAR PROPERTY FOR SYMMETRIC ALGEBRAS
}

\author{
FLORIAN EISELE, MICHAEL GELINE, RADHA KESSAR, AND MARKUS LINCKELMANN
}

\begin{abstract}
We identify a class of symmetric algebras over a complete discrete valuation ring $\mathcal{O}$ of characteristic zero to which the characterisation of Knörr lattices in terms of stable endomorphism rings in the case of finite group algebras, can be extended. This class includes finite group algebras, their blocks and source algebras and Hopf orders. We also show that certain arithmetic properties of finite group representations extend to this class of algebras. Our results are based on an explicit description of Tate duality for lattices over symmetric $\mathcal{O}$-algebras whose extension to the quotient field of $\mathcal{O}$ is separable.
\end{abstract}

\section{INTRODUCTION}

Let $p$ be a prime. Let $\mathcal{O}$ be a complete discrete valuation ring with maximal ideal $J(\mathcal{O})=\pi \mathcal{O}$ for some $\pi \in \mathcal{O}$, residue field $k=\mathcal{O} / J(\mathcal{O})$ of characteristic $p$, and field of fractions $K$ of characteristic zero. An $\mathcal{O}$-algebra $A$ is symmetric if $A$ is isomorphic to its $\mathcal{O}$-dual $A^{*}$ as an $A$ - $A$-bimodule; this implies that $A$ is free of finite rank over $\mathcal{O}$. The image $s$ of $1_{A}$ under a bimodule isomorphism $A \cong$ $A^{*}$ is called a symmetrising form for $A$; it has the property that $s(a b)=s(b a)$ for all $a, b \in A$ and that the bimodule isomorphism $A \cong A^{*}$ sends $a \in A$ to the map $s_{a} \in A^{*}$ defined by $s_{a}(b)=s(a b)$ for all $a, b \in A$. Since the automorphism group of $A$ as an $A$-A-bimodule is canonically isomorphic to $Z(A)^{\times}$, any other symmetrising form of $A$ is of the form $s_{z}$ for some $z \in Z(A)^{\times}$. If $X$ is an $\mathcal{O}$-basis of $A$, then any symmetrising form $s$ of $A$ determines a dual basis $X^{\vee}=\left\{x^{\vee} \mid x \in X\right\}$ satisfying $s\left(x x^{\vee}\right)=1$ for $x \in X$ and $s\left(x y^{\vee}\right)=0$ for $x, y \in X, x \neq y$. We denote by $\operatorname{Tr}_{1}^{A}: A \rightarrow$ $Z(A)$ the $Z(A)$-linear map defined by $\operatorname{Tr}_{1}^{A}(a)=\sum_{x \in X} x a x^{\vee}$ for all $a \in A$. This map depends on the choice of $s$ but not on the choice of the basis $X$. We set $z_{A}=\operatorname{Tr}_{1}^{A}\left(1_{A}\right)$ and call $z_{A}$ the relative projective element of $A$ in $Z(A)$ with respect to $s$. This is also called the central Casimir element in 4. If $z \in Z(A)^{\times}$and $s^{\prime}=s_{z}$, then the dual basis of $X$ with respect to $s^{\prime}$ is equal to $X^{\vee} z^{-1}$, where $X^{\vee}$ is the dual basis of $X$ with respect to $s$, and hence the relatively projective element in $Z(A)$ with respect to $s^{\prime}$ is equal to $z_{A}^{\prime}=z_{A} z^{-1}$. If we do not specify a symmetric form of a symmetric algebra $A$, then the relative projective elements form a $Z(A)^{\times}$-orbit in $Z(A)$. See Broué [4] for more details.

The purpose of this paper is to examine situations in which some relative projective element is a scalar multiple of the identity.

Definition 1.1. A symmetric $\mathcal{O}$-algebra $A$ is said to have the projective scalar property if there exists a symmetrising form $s$ of $A$ such that the corresponding relative projective element $z_{A}$ is of the form $z_{A}=\lambda 1_{A}$ for some $\lambda \in \mathcal{O}$.

2010 Mathematics Subject Classification. Primary 20C20; Secondary 16H10. 
Throughout the paper we will be working with a symmetric $\mathcal{O}$-algebra $A$ such that the $K$ algebra $K \otimes_{\mathcal{O}} A$ is separable. Since $K$ has characteristic zero, $K \otimes_{\mathcal{O}} A$ is separable if and only if it is semisimple. This in turn is equivalent to the condition that the relative projective element with respect to some, and hence any, symmetrising form on $A$ is invertible in $Z\left(K \otimes_{\mathcal{O}} A\right)$ (see 4 , Proposition 3.6]). In particular, in case $A$ has the projective scalar property, the separability of $K \otimes_{\mathcal{O}} A$ is equivalent to the property that the relative projective elements of $A$ are non-zero.

Matrix algebras, finite group algebras, blocks and source algebras of finite group algebras, as well as Hopf algebras whose extension to $K$ is semisimple have the projective scalar property (see Examples 5.1, 5.2. and 5.3), but Iwahori-Hecke algebras and rings of generalized characters do not typically have this property (see Examples 5.4, 5.5, and 5.6). The projective scalar property is invariant under taking direct factors and tensor products but not under direct products, and is not invariant under Morita equivalences (see Example 5.1).

Our motivation for studying algebras with the projective scalar property comes from a characterisation of Knörr lattices for a finite group algebra in terms of the relatively $\mathcal{O}$-stable module category of the algebra. Recall that an $A$-lattice is a left unital $A$-module which is free of finite

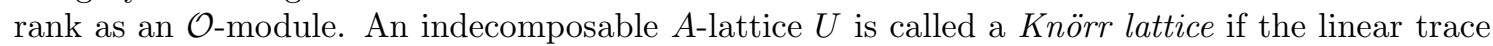
form $\operatorname{tr}_{U}$ on $\operatorname{End}_{\mathcal{O}}(U)$ satisfies $\operatorname{tr}_{U}(\alpha) \mathcal{O} \subseteq \operatorname{rk}_{\mathcal{O}}(U) \mathcal{O}$ for every $\alpha \in \operatorname{End}_{A}(U)$, with equality precisely when $\alpha$ is an automorphism.

Now for two finitely generated $A$-modules $U$ and $V$, we denote by $\underline{\operatorname{Hom}}_{A}(U, V)$ the homomorphism space in the $\mathcal{O}$-stable category $\underline{\bmod }(A)$ of finitely generated $A$-modules; that is, $\underline{\operatorname{Hom}}_{A}(U, V)$ is the quotient of $\operatorname{Hom}_{A}(U, V)$ by the subspace $\operatorname{Hom}_{A}^{\mathrm{pr}}(U, V)$ of $A$-homomorphisms $U \rightarrow V$ which factor through a relatively $\mathcal{O}$-projective $A$-module. We write $\operatorname{End}_{A}^{\mathrm{pr}}(U)=\operatorname{Hom}_{A}^{\mathrm{pr}}(U, U)$ and $\underline{\operatorname{End}}_{A}(U)=\underline{\operatorname{Hom}}_{A}(U, U)$.

For an $A$-lattice $U$, let $a(U)$ denote the smallest non-negative integer such that $\pi^{a(U)}$ annihilates End $_{A}(U)$. In [6], the element $\pi^{a(U)}$ is referred to as the exponent of $U$. If $U$ is indecomposable non-projective, $U$ is said to have the stable exponent property if the socle of $\underline{\text { End }}_{A}(U)$ as a (left or right) module over itself is equal to $\pi^{a(U)-1} \underline{\operatorname{End}}_{A}(U)$.

Carlson and Jones [6], and independently Thevenaz [19] and Knörr [10] proved that for $G$ a finite group, an absolutely indecomposable non-projective $\mathcal{O} G$-lattice is a Knörr lattice if and only if it has the stable exponent property. The projective scalar property guarantees such an equivalence:

Theorem 1.2. Let $A$ be a symmetric $\mathcal{O}$-algebra such that $K \otimes_{\mathcal{O}} A$ is separable. Suppose that $A$ has the projective scalar property. Then an indecomposable non-projective A-lattice $U$ is a Knörr lattice if and only if $U$ is absolutely indecomposable and has the stable exponent property.

The converse to this theorem is false. In Example 5.8, we shall see a symmetric algebra without the projective scalar property for which the Knörr lattices coincide with those having the stable exponent property. Thus, the equivalence between the Knörr and stable exponent properties does not provide a characterization of the projective scalar property. Also, in Example 5.7 we shall see both Knörr lattices which do not have the stable exponent property, as well as lattices with the stable exponent property which are not Knörr.

Example 5.7 will, in addition, show that the property of being a Knörr lattice is not invariant under Morita equivalences. However, it is easy to see that the stable exponent property is invariant under such equivalences. Thus, two subclasses can be identified within a given Morita equivalence class of symmetric algebras: namely, those for which the above two types of lattices coincide, and those with the projective scalar property. 
The basic ingredient for the proof of Theorem 1.2 is a description of Tate duality for lattices over symmetric $\mathcal{O}$-algebras with separable coefficient extensions which makes the role of the relative projective element explicit. Note that $\underline{\operatorname{Hom}}_{A}(U, V)$ is a torsion $\mathcal{O}$-module for any $A$-lattices $U$ and $V$ when $K \otimes_{\mathcal{O}} A$ is separable. This follows from the Gaschütz-Ikeda Lemma (cf. [8, Lemma 7.1.11]), which is a special case of Higman's criterion for modules over symmetric algebras in Broué [4].

Theorem 1.3. Let $A$ be a symmetric $\mathcal{O}$-algebra with symmetrising form s such that $K \otimes_{\mathcal{O}} A$ is separable. Set $z=z_{A}$. Let $U, V$ be A-lattices. The map sending $(\alpha, \beta) \in \operatorname{Hom}_{A}(U, V) \times$ $\operatorname{Hom}_{A}(V, U)$ to $\operatorname{tr}_{K \otimes_{\mathcal{O}} U}\left(z^{-1} \beta \circ \alpha\right) \in K$ induces a non degenerate pairing

$$
\underline{\operatorname{Hom}}_{A}(U, V) \times \underline{\operatorname{Hom}}_{A}(V, U) \rightarrow K / \mathcal{O} .
$$

Here $\operatorname{tr}_{K \otimes_{\mathcal{O}} U}\left(z^{-1} \beta \circ \alpha\right)$ is the trace of the $K$-linear endomorphism of $K \otimes_{\mathcal{O}} U$ obtained from extending the endomorphism $\beta \circ \alpha$ of $U$ linearly to $K \otimes_{\mathcal{O}} U$, composed with the endomorphism given by multiplication on $K \otimes_{\mathcal{O}} U$ with the inverse $z^{-1}$ of $z$ in $Z\left(K \otimes_{\mathcal{O}} A\right)$. If $A$ has the projective scalar property, then the Tate duality pairing admits the following description (which is in this form well-known for finite group algebras; see [5, Theorem (7.4)]).

Corollary 1.4. Let $A$ be a symmetric $\mathcal{O}$-algebra such that $K \otimes_{\mathcal{O}} A$ is separable. Suppose that $z_{A}=\pi^{n} 1_{A}$ for some choice of a symmetrising form of $A$ and some positive integer $n$. Let $U$ and $V$ be A-lattices. The map sending $(\alpha, \beta) \in \operatorname{Hom}_{A}(U, V) \times \operatorname{Hom}_{A}(V, U)$ to $\operatorname{tr}_{U}(\beta \circ \alpha)$ induces a non degenerate pairing

$$
\underline{\operatorname{Hom}}_{A}(U, V) \times \underline{\operatorname{Hom}}_{A}(V, U) \rightarrow \mathcal{O} / \pi^{n} \mathcal{O} .
$$

Remark 1.5. Theorem 1.3, applied to $U=V$, shows that if $U$ is an indecomposable nonprojective lattice for a symmetric $\mathcal{O}$-algebra $A$ such that $K \otimes_{\mathcal{O}} A$ is separable, then the socle of $\underline{\operatorname{End}}_{A}(U)$ as a module over itself is simple, since it is dual to $\underline{\text { End }}_{A}(U) / J\left(\underline{\operatorname{End}}_{A}(U)\right) \cong k$. This fact is well-known - see Roggenkamp [18] - and this is the key step in the existence proof of almost split sequences of $A$-modules. Applying Theorem 1.3 to Heller translates of $V$ yields non degenerate pairings

$$
\widehat{\operatorname{Ext}}_{A}^{n}(U, V) \times \widehat{\operatorname{Ext}}_{A}^{-n}(V, U) \rightarrow K / \mathcal{O}
$$

for any integer $n$. Applied to $U=V=A$ as a module over $A \otimes_{\mathcal{O}} A^{\text {op }}$ this yields non degenerate pairings in Tate-Hochschild cohomology

$$
\widehat{H H}^{n}(A) \times \widehat{H H}^{-n}(A) \rightarrow K / \mathcal{O} .
$$

Theorem 1.2 is a special case of the following consequence of Theorem 1.3 which gives a characterisation of absolutely indecomposable modules with the stable exponent property for symmetric $\mathcal{O}$-algebras. Denote by $\nu$ a $\pi$-adic valuation on $K$.

Theorem 1.6. Let $A$ be a symmetric $\mathcal{O}$-algebra with symmetrising form s such that $K \otimes_{\mathcal{O}} A$ is separable. Denote by $z$ the associated relatively projective element of $A$ in $Z(A)$. Let $U$ be an indecomposable non projective A-lattice. The following are equivalent.

(i) For any $\alpha \in \operatorname{End}_{A}(U)$ we have $\nu\left(\operatorname{tr}_{K \otimes_{\mathcal{O}} U}\left(z^{-1} \alpha\right)\right) \geq \nu\left(\operatorname{tr}_{K \otimes_{\mathcal{O}} U}\left(z^{-1} \operatorname{Id}_{U}\right)\right)$, with equality if and only if $\alpha$ is an automorphism of $U$.

(ii) The A-lattice $U$ is absolutely indecomposable and has the stable exponent property.

Symmetric $\mathcal{O}$-algebras with split semisimple coefficient extensions to $K$ having the projective scalar property can be characterised as follows. 
Theorem 1.7. Let $A$ be a symmetric $\mathcal{O}$-algebra such that $K \otimes_{\mathcal{O}} A$ is split semisimple. Denote by $\rho: A \rightarrow \mathcal{O}$ the regular character of $A$. The following are equivalent.

(i) The algebra $A$ has the projective scalar property.

(ii) There exists a non-negative integer $n$ such that $\pi^{-n} \rho$ is a symmetrising form of $A$.

(iii) There exists a non-negative integer $n$ such that for any A-lattice $U$ we have

$$
\operatorname{tr}_{U}\left(\operatorname{End}_{A}(U)\right)=\pi^{n-a(U)} \mathcal{O} .
$$

Moreover, if these three equivalent statements hold, then the integers $n$ in (ii) and (iii) coincide, and $\pi^{n} 1_{A}$ is a relative projective element with respect to some symmetrising form of $A$.

We also have a characterisation, in terms of the decomposition matrix, of symmetric $\mathcal{O}$-algebras $A$ such that some algebra in the Morita or derived equivalence class of $A$ has the scalar projective property. Recall that if $B$ is a split finite dimensional algebra over a field $F$ then the set of characters of simple $A$-modules is a linearly independent subset of the $F$-vector space of functions from $B$ to $F$ (see for instance [16. Chapter 3, Theorem 3.13]), and hence may be identified with a set of representatives of the isomorphism classes of simple $B$-modules.

Theorem 1.8. Let $A$ be a symmetric $\mathcal{O}$-algebra such that $K \otimes_{\mathcal{O}} A$ is split semisimple and $k \otimes_{\mathcal{O}} A$ is split. Denote by $\operatorname{Irr}_{K}(A)$ the set of characters of simple $K \otimes_{\mathcal{O}} A$ modules and by $\operatorname{Irr}_{k}(A)$ the set of characters of simple $k \otimes_{\mathcal{O}} A$-modules. For $\chi \in \operatorname{Irr}_{K}(A)$ and $\varphi \in \operatorname{Irr}_{k}(A)$ denote by $d_{\chi, \varphi}$ the multiplicity of $S$ as a composition factor of $k \otimes_{\mathcal{O}} V$, where $V$ is an A-lattice such that $K \otimes_{\mathcal{O}} V$ has character $\chi$, and $S$ is a simple $k \otimes_{\mathcal{O}} A$-module with character $\varphi$. The following are equivalent.

(i) There exists an algebra Morita equivalent to $A$ with the projective scalar property.

(ii) There exists an algebra derived equivalent to A with the projective scalar property.

(iii) There exists a non-negative integer $n$ and positive integers $m_{\varphi}, \varphi \in \operatorname{Irr}_{k}(A)$ such that setting $a_{\chi}:=\sum_{\varphi \in \operatorname{Irr}_{k}(A)} m_{\varphi} d_{\chi, \varphi}, \chi \in \operatorname{Irr}_{K}(A)$, the form $\pi^{-n} \sum_{\chi \in \operatorname{Irr}_{K}(A)} a_{\chi} \chi$ is a symmetrising form for $A$.

(iv) There exists a non-negative integer $n$ and integers $m_{\varphi}, \varphi \in \operatorname{Irr}_{k}(A)$ such that setting $a_{\chi}:=\sum_{\varphi \in \operatorname{Irr}_{k}(A)} m_{\varphi} d_{\chi, \varphi}, \chi \in \operatorname{Irr}_{K}(A)$, the form $\pi^{-n} \sum_{\chi \in \operatorname{Irr}_{K}(A)} a_{\chi} \chi$ is a symmetrising form for $A$.

We point out that certain arithmetic features of finite group representations carry over to algebras with the projective scalar property. Recall that the degree of an ordinary irreducible character of a finite group $G$ divides the order of $G$ and that if $U$ is a projective $\mathcal{O} G$-lattice, then the $p$-part of $|G|$ divides the $p$-part of the $\mathcal{O}$-rank of $U$.

Proposition 1.9. Let $A$ be a symmetric $\mathcal{O}$-algebra such that $K \otimes_{\mathcal{O}} A$ is split semisimple. Assume that $A$ has the projective scalar property and let $\pi^{n} 1_{A}$ be a relative projective element with respect to some symmetrizing form on A.

(i) If $U$ is a Knörr A-lattice, then the p-part of the $\mathcal{O}$-rank of $U$ divides $\pi^{n}$ in $\mathcal{O}$.

(ii) If $U$ is a projective $A$-lattice, then the p-part of the $\mathcal{O}$-rank of $U$ is divisible in $\mathcal{O}$ by $\pi^{n}$.

Remark 1.10. Note that if $A=\mathcal{O} G$, then $|G| \cdot 1_{\mathcal{O} G}$ is the relative projective element with respect to the standard symmetrising form (see [4, Examples and Remarks after Proposition 3.3]). Moreover, an absolutely irreducible $\mathcal{O} G$-lattice is a Knörr $\mathcal{O} G$-lattice. Hence, letting $p$ vary across all primes in (i), one sees that the above does generalise the corresponding results for group algebras. A related global divisbility criterion for irreducible lattices of symmetric algebras has been given by Jacoby and Lorenz [14, Corollary 6] in the context of Kaplansky's sixth conjecture. 
For $U$ an $A$-lattice, define the height of $U$ to be the number $h(U)$ such that

$$
\operatorname{rank}(U)_{p}=p^{m+h(U)},
$$

where $m$ is defined by

$$
p^{m}=\min _{V}\left\{\operatorname{rank}(V)_{p}\right\}
$$

as $V$ ranges over all irreducible $A$-lattices. Note that $h(U)$ is a non-negative integer.

It is well known that a Morita equivalence between blocks of finite group algebras or between a block algebra and the corresponding source algebra preserves the height of corresponding irreducible characters (see [2] and [3]). The following theorem generalises this to algebras with the projective scalar property and to Knörr lattices.

Theorem 1.11. Suppose that $K \otimes_{\mathcal{O}} A$ is split semisimple. Let $A^{\prime}$ be an $\mathcal{O}$-algebra Morita equivalent to $A$, and suppose that both $A$ and $A^{\prime}$ have the projective scalar property. Let $U$ be a Knörr $A$ lattice and let $U^{\prime}$ be an $A^{\prime}$-lattice corresponding to $U$ through a Morita equivalence between $A$ and $A^{\prime}$. Then $U^{\prime}$ is a Knörr $A^{\prime}$-lattice and $h(U)=h\left(U^{\prime}\right)$.

Finally we point out that although the stable exponent property does not apply to projective lattices, we can, following Knörr [9, Lemma 1.9], characterise projective Knörr lattices in the presence of the projective scalar property.

Proposition 1.12. Let $A$ be as in the previous proposition. Assume that $U$ is an A-lattice which is both projective and Knörr. Then $U / \pi U$ is a simple $A / \pi A$-module. In particular, $K \otimes_{\mathcal{O}} U$ is an irreducible $K \otimes_{\mathcal{O}}$ A-module.

Section 2 contains the proof of Theorem 1.3 Theorem 1.6 and Theorem 1.2 We prove Theorems 1.7 and 1.8 in Section 3. This section also contains a characterisation of the projective scalar property in terms of rational centres. Section 4 discusses arithmetic properties of Knörr lattices in the presence of the scalar projective property, including the proof of Proposition 1.9 and Theorem 1.11 Section 5 contains various examples.

\section{Tate Duality for Symmetric algebras}

The proof of Theorem 1.3 is an adaptation of ideas in Thévenaz [19, Section 1]. We keep the notation in Theorem 1.3. For simplicity, we write in this section $K A=K \otimes_{\mathcal{O}} A, K U=K \otimes_{\mathcal{O}} U$, and $K V=K \otimes_{\mathcal{O}} V$. We write $K \operatorname{Hom}_{\mathcal{O}}(U, V)=K \otimes_{\mathcal{O}} \operatorname{Hom}_{\mathcal{O}}(U, V)$ and identify this space with $\operatorname{Hom}_{K}(K U, K V)$ whenever convenient. Similarly, we write $K \operatorname{Hom}_{A}(U, V)=K \otimes_{\mathcal{O}} \operatorname{Hom}_{A}(U, V)$ and identify this space with $\operatorname{Hom}_{K A}(K U, K V)$. Let $X, X^{\vee}$ be a pair of $\mathcal{O}$-bases of $A$ dual to each other with respect to the symmetrising form $s$; in particular, the relative projective element with respect to $s$ is

$$
z_{A}=\sum_{x \in X} x x^{\vee}=\sum_{x \in X} x^{\vee} x
$$

where $x^{\vee}$ denotes the unique element in $X^{\vee}$ satisfying $s\left(x x^{\vee}\right)=1$, for $x \in X$. We denote by

$$
\operatorname{Tr}_{1}^{A}: K \operatorname{Hom}_{\mathcal{O}}(U, V) \rightarrow K \operatorname{Hom}_{A}(U, V)
$$

the $K$-linear map sending $\alpha \in \operatorname{Hom}_{\mathcal{O}}(U, V)$ to $\sum_{x \in X} x \alpha x^{\vee}$. Here $x \alpha x^{\vee} \in \operatorname{Hom}_{\mathcal{O}}(U, V)$ is defined by $\left(x \alpha x^{\vee}\right)(u)=x \alpha\left(x^{\vee} u\right)$ for $u \in U$ and $x \in X$. Clearly, $\operatorname{Tr}_{1}^{A}$ restricts to a map $\operatorname{Hom}_{\mathcal{O}}(U, V) \rightarrow$ 
$\operatorname{Hom}_{A}(U, V)$. By Higman's criterion for symmetric algebras (cf. [4]), we have $\operatorname{Tr}_{1}^{A}\left(\operatorname{Hom}_{\mathcal{O}}(U, V)\right)=$ $\operatorname{Hom}_{A}^{\mathrm{pr}}(U, V)$. Denote by

$$
\varphi: K \operatorname{Hom}_{\mathcal{O}}(U, V) \times K \operatorname{Hom}_{\mathcal{O}}(V, U) \rightarrow K
$$

the $K$-linear map sending $(\alpha, \beta) \in \operatorname{Hom}_{\mathcal{O}}(U, V) \times \operatorname{Hom}_{\mathcal{O}}(V, U)$ to $\operatorname{tr}_{U}(\beta \circ \alpha)$, and denote by

$$
\varphi_{A}: K \operatorname{Hom}_{A}(U, V) \times K \operatorname{Hom}_{A}(V, U) \rightarrow K
$$

the map sending $(\alpha, \beta) \in \operatorname{Hom}_{A}(U, V) \times \operatorname{Hom}_{A}(V, U)$ to $\operatorname{tr}_{K U}\left(z_{A}^{-1} \beta \circ \alpha\right)$, where $\alpha, \beta$ are extended linearly to maps between $K U, K V$. The following fact generalises [19, Prop. 1.1].

Proposition 2.1. With the notation above, for $\alpha \in \operatorname{Hom}_{\mathcal{O}}(U, V)$ and $\beta \in \operatorname{Hom}_{A}(V, U)$ we have

$$
\varphi_{A}\left(\operatorname{Tr}_{1}^{A}(\alpha), \beta\right)=\varphi(\alpha, \beta) .
$$

Similarly, for $\gamma \in \operatorname{Hom}_{A}(U, V)$ and $\delta \in \operatorname{Hom}_{\mathcal{O}}(V, U)$ we have

$$
\varphi_{A}\left(\gamma, \operatorname{Tr}_{1}^{A}(\delta)\right)=\varphi(\gamma, \delta) \text {. }
$$

In particular, $\varphi_{A}$ is non-degenerate.

Proof. We regard $\operatorname{Hom}_{\mathcal{O}}(U, V)$ and $\operatorname{Hom}_{\mathcal{O}}(V, U)$ as $A$-A-bimodules in the canonical way. If $\mu \in$ $\operatorname{Hom}_{\mathcal{O}}(U, V)$ and $\beta \in \operatorname{Hom}_{\mathcal{O}}(V, U)$, then for any $a \in A$, we have $\beta \circ a \mu=\beta a \circ \mu$. If $\epsilon \in \operatorname{End}_{\mathcal{O}}(U)$ and $a \in A$, then $\operatorname{tr}_{U}(\epsilon a)=\operatorname{tr}_{U}(a \epsilon)$. Thus we have

$$
\begin{aligned}
\varphi_{A}\left(\operatorname{Tr}_{1}^{A}(\alpha), \beta\right) & =\operatorname{tr}_{K U}\left(z_{A}^{-1} \sum_{x \in X} \beta \circ x \alpha x^{\vee}\right)=\operatorname{tr}_{K U}\left(z_{A}^{-1} \sum_{x \in X} x^{\vee} \beta \circ x \alpha\right) \\
& =\operatorname{tr}_{K U}\left(z_{A}^{-1} \sum_{x \in X} x^{\vee} \beta x \circ \alpha\right)=\operatorname{tr}_{K U}\left(z_{A}^{-1} \sum_{x \in X} x^{\vee} x \beta \circ \alpha\right) \\
& =\operatorname{tr}_{K U}\left(z_{A}^{-1} z_{A} \beta \circ \alpha\right)=\varphi(\alpha, \beta) .
\end{aligned}
$$

This shows the first equality, and the proof of the second is analogous. Clearly $\varphi$ is non degenerate, and hence so is $\varphi_{A}$.

Proof of Theorem 1.3. For $E$ an $\mathcal{O}$-submodule of $\operatorname{Hom}_{K A}(K U, K V)$ denote by $E^{\perp}$ the $\mathcal{O}$-submodule in $\operatorname{Hom}_{K A}(K V, K U)$ consisting of all $\beta \in \operatorname{Hom}_{K A}(K V, K U)$ such that $\varphi_{A}(\epsilon, \beta) \in \mathcal{O}$ for all $\epsilon \in E$. By the previous proposition, $\varphi_{A}$ is non degenerate, and hence if $E$ is a lattice in $\operatorname{Hom}_{K A}(K U, K V)$, then $E^{\perp}$ is a lattice in $\operatorname{Hom}_{K A}(K V, K U)$, and we have $\left(E^{\perp}\right)^{\perp}=E$. We need to show that $\left(\operatorname{Hom}_{A}^{\mathrm{pr}}(U, V)\right)^{\perp}=\operatorname{Hom}_{A}(V, U)$. Let $\beta \in \operatorname{Hom}_{K A}(K U, K V)$. We have $\beta \in\left(\operatorname{Hom}_{A}^{\mathrm{pr}}(U, V)\right)^{\perp}$ if and only if $\varphi_{A}\left(\operatorname{Tr}_{1}^{A}(\alpha), \beta\right) \in \mathcal{O}$ for all $\alpha \in \operatorname{Hom}_{\mathcal{O}}(U, V)$. By Proposition 2.1, this is equivalent to $\operatorname{tr}_{K U}(\beta \circ \alpha) \in \mathcal{O}$ for all $\alpha \in \operatorname{Hom}_{\mathcal{O}}(U, V)$. This, in turn, is the case if and only if $\beta$ belongs to the subspace $\operatorname{Hom}_{A}(U, V)$ of $\operatorname{Hom}_{K A}(K U, K V)$. (To see this, choose a basis of $U$, a basis of $V$, and let $\alpha$ range over the maps sending exactly one basis element in $U$ to a basis element in $V$ and all other basis elements of $U$ to 0 ).

Proof of Corollary 1.4. We have $z_{A}=\pi^{n} 1_{A}$. The non degenerate pairing

$$
\underline{\operatorname{Hom}}_{A}(U, V) \times \underline{\operatorname{Hom}}_{A}(V, U) \rightarrow K / \mathcal{O}
$$

from Theorem 1.3 has image contained in the submodule $\pi^{-n} \mathcal{O} / \mathcal{O}$ of $K / \mathcal{O}$. Multiplication by $\pi^{n}$ yields an isomorphism $\pi^{-n} \mathcal{O} / \mathcal{O} \cong \mathcal{O} / \pi^{n} \mathcal{O}$. Thus Corollary 1.4 follows from Theorem 1.3.

In order to prove Theorem [1.6, we need the following generalisation of [6, Prop. 4.2]. 
Proposition 2.2. Let $A$ be a symmetric $\mathcal{O}$-algebra with symmetrising form s such that $K \otimes_{\mathcal{O}} A$ is separable. Set $z=z_{A}$. Let $U$ be an A-lattice and let a be the smallest non-negative integer such that $\pi^{a}$ annihilates $\underline{\text { End }}_{A}(U)$. Then

$$
\pi^{a} \operatorname{tr}_{K U}\left(z^{-1} \operatorname{End}_{A}(U)\right)=\mathcal{O} .
$$

Proof. Let $\alpha \in \operatorname{End}_{A}(U)$. By the assumptions we have $\pi^{a} \alpha \in \operatorname{End}_{A}^{\mathrm{pr}}(U)$. Theorem 1.3, applied with $U=V$ and $\beta=\operatorname{Id}_{U}$ implies that $\pi^{a} \operatorname{tr}_{K U}\left(z^{-1} \alpha\right) \in \mathcal{O}$. Thus $\pi^{a} \operatorname{tr}_{K U}\left(z^{-1} \operatorname{End}_{A}(U)\right) \subseteq \mathcal{O}$. For the reverse inclusion, consider first the case that $U$ is non-projective. Then $a \geq 1$, and $\pi^{a-1} \mathrm{Id}_{U}$ is not contained in $\operatorname{End}_{A}^{\mathrm{pr}}(U)$; equivalently, its image in $\underline{\operatorname{End}}_{A}(U)$ is nonzero. Again by Theorem 1.3. there exists $\alpha \in \operatorname{End}_{A}(U)$ such that $\pi^{a-1} \operatorname{tr}_{U}\left(z^{-1} \alpha\right) \notin \mathcal{O}$. Thus $\pi^{a} \operatorname{tr}_{K U}\left(z^{-1} \operatorname{End}_{A}(U)\right)$ is not contained in $\pi \mathcal{O}$, whence the equality in this case. Suppose $U$ is projective, so $a=0$. Let $\alpha \in$ $\operatorname{End}_{\mathcal{O}}(U)$ be such that $\left(\operatorname{tr}_{U}(\alpha)\right)=1$ and set $\beta=\operatorname{Tr}_{1}^{A}(\alpha) \in \operatorname{End}_{A}(U)$. By Proposition 2.1, we have $\operatorname{tr}_{U}\left(z^{-1} \beta\right)=\varphi_{A}\left(\operatorname{Tr}_{1}^{A}(\alpha), \operatorname{Id}_{U}\right)=\varphi\left(\alpha, \operatorname{Id}_{U}\right)=\operatorname{tr}_{U}(\alpha)=1$. The result follows.

Proof of Theorem [1.6. Let $a$ be the smallest positive integer such that $\pi^{a}$ annihilates $\underline{E n d}_{A}(U)$. The algebra $\underline{\operatorname{End}}_{A}(U)$ is local, as $U$ is indecomposable non projective. The duality in Theorem 1.3 implies that $\operatorname{soc}\left(\underline{\operatorname{End}}_{A}(U)\right)$ is simple.

Suppose that (i) holds. We show first that $U$ is absolutely indecomposable. The inequality in (i) applied to the endomorphism $\alpha$ given by multiplication with $z$ shows that

$$
\nu\left(\operatorname{rk}_{\mathcal{O}}(U)\right)=\nu\left(\operatorname{tr}_{U}\left(\operatorname{Id}_{U}\right)\right) \geq \nu\left(\operatorname{tr}_{K U}\left(z^{-1} \operatorname{Id}_{U}\right)\right),
$$

so in particular, $\operatorname{tr}_{K U}\left(z^{-1} \operatorname{Id}_{U}\right)$ is nonzero. The inequality in (i) applied to an arbitrary $\alpha \in$ $\operatorname{End}_{A}(U)$ implies that the scalar $\tau$ defined by

$$
\tau=\operatorname{tr}_{K U}\left(z^{-1} \alpha\right) \operatorname{tr}_{K U}\left(z^{-1} \operatorname{Id}_{U}\right)^{-1}
$$

belongs to $\mathcal{O}$. A trivial verification shows that

$$
\operatorname{tr}_{K U}\left(z^{-1}\left(\alpha-\tau \operatorname{Id}_{U}\right)\right)=0 .
$$

Thus condition (i) implies that $\alpha-\tau \operatorname{Id}_{U}$ is not an automorphism, hence in $J\left(\operatorname{End}_{A}(U)\right)$. It follows that $\operatorname{End}_{A}(U)=\mathcal{O} \cdot \operatorname{Id}_{U}+J\left(\operatorname{End}_{A}(U)\right)$, and hence $U$ is absolutely indecomposable.

We show next that $U$ has the stable exponent property. Since the socle of $\underline{\operatorname{End}}_{A}(U)$ is simple, we have $\operatorname{soc}\left(\underline{\operatorname{End}}_{A}(U)\right) \subseteq \pi^{a-1} \underline{\operatorname{End}}_{A}(U)$, and it suffices therefore to show that $\pi^{a-1} \underline{\operatorname{End}}_{A}(U)$ is a semisimple End ${ }_{A}(U)$-module. That is, it suffices to show that $\pi^{a-1}$ End $_{A}(U)$ is annihilated by $J\left(\underline{\operatorname{End}}_{A}(U)\right)$. Let $\alpha \in J\left(\operatorname{End}_{A}(U)\right)$. The assumptions in (i) together with Proposition 2.2 imply that $\pi^{a} \operatorname{tr}_{K U}\left(z^{-1} \alpha\right) \in \pi \mathcal{O}$, hence $\pi^{a-1} \operatorname{tr}_{K U}\left(z^{-1} \alpha\right) \in \mathcal{O}$. By Theorem 1.3 this is equivalent to $\pi^{a-1} \alpha \in \operatorname{End}_{A}^{\mathrm{pr}}(U)$, or equivalently, to $\pi^{a-1} \underline{\alpha}=0$. This shows that (i) implies (ii).

Suppose conversely that (ii) holds. In particular, the socle of $\operatorname{End}_{A}(U)$ is simple and equal to $\pi^{a-1} \underline{\operatorname{End}}_{A}(U)$. Let $\alpha \in J\left(\operatorname{End}_{A}(U)\right)$. The image $\underline{\alpha}$ in $\underline{\operatorname{End}}_{A}(U)$ is contained in $J\left(\underline{\operatorname{End}}_{A}(U)\right)$, and hence $\underline{\alpha}$ annihilates $\pi^{a-1} \underline{\operatorname{End}}_{A}(U)$. Thus $\pi^{a-1} \underline{\alpha}=0$. Theorem 1.3 implies that $\pi^{a-1} \operatorname{tr}_{K U}\left(z^{-1} \alpha\right) \in$ $\mathcal{O}$, hence $\pi^{a} \operatorname{tr}_{K U}\left(z^{-1} \alpha\right) \in \pi \mathcal{O}$.

By Proposition 2.2 there exists $\alpha \in \operatorname{End}_{A}(U)$ such that $\pi^{a} \operatorname{tr}_{K U}\left(z^{-1} \alpha\right)=1$. By the previous argument, this forces $\alpha \notin J\left(\operatorname{End}_{A}(U)\right)$. Since $U$ is absolutely indecomposable, it follows that $\operatorname{End}_{A}(U)$ is split local, and hence we have $\alpha=\lambda \operatorname{Id}_{U}+\rho$ for some $\lambda \in \mathcal{O}^{\times}$and some $\rho \in$ $J\left(\operatorname{End}_{A}(U)\right)$. Since $\pi^{a} \operatorname{tr}_{K U}\left(z^{-1} \rho\right) \in \pi \mathcal{O}$, it follows that $\pi^{a} \operatorname{tr}_{K U}\left(z^{-1} \lambda \operatorname{Id}_{U}\right) \in \mathcal{O}^{\times}$. Then in fact $\pi^{a} \operatorname{tr}_{K U}\left(z^{-1} \lambda \operatorname{Id}_{U}\right) \in \mathcal{O}^{\times}$for any $\lambda \in \mathcal{O}^{\times}$, and hence $\pi^{a} \operatorname{tr}_{K U}\left(z^{-1} \alpha\right) \in \mathcal{O}^{\times}$for any automorphism $\alpha$ of $U$. This shows that (ii) implies (i). 
Proof of Theorem 1.2. Let $n$ be the positive integer such that $z_{A}=\pi^{n} 1_{A}$, for some choice of a symmetrising form. Condition (i) in Theorem 1.6 is then equivalent to stating that $U$ is a Knörr lattice. Thus Theorem 1.2 follows from Theorem 1.6

\section{Characterisations of the Projective SCAlar Property}

Throughout this section, $A$ will denote an $\mathcal{O}$-order such that $K \otimes_{\mathcal{O}} A$ is separable. We identify $A$ with its canonical image in $K A=K \otimes_{\mathcal{O}} A$. Denote by $\operatorname{Irr}_{K}(A)$ the set of the characters of the simple $K A$-modules. For $\chi \in \operatorname{Irr}_{K}(A)$ denote by $e(\chi)$ the unique primitive idempotent in $Z(K A)$ satisfying $\chi(e(\chi)) \neq 0$. We will use this notation for other orders as well.

Proof of Theorem 1.7. Suppose that $K \otimes_{\mathcal{O}} A$ is split semisimple. Proposition 2.2 shows that (i) implies (iii).

By the assumptions, $K A e(\chi)$ is a matrix algebra over $K$ of dimension $\chi(1)^{2}$. In particular, $K A e(\chi)$ is symmetric with symmetrising form $\chi$, and we have $Z(K A)=\prod_{\chi \in \operatorname{Irr}_{K}(A)} K e(\chi)$. Fix a symmetrising form $s$ of $A$. Then $s$ extends to a symmetrising form of $K A$, still denoted $s$, and we have

$$
s=\sum_{\chi \in \operatorname{Irr}_{K}(A)} \sigma_{\chi} \cdot \chi
$$

for some $\sigma_{\chi} \in K$. The relative projective element of the matrix algebra $K A e(\chi)$ with respect to $\chi$ is $\chi(1) \cdot e(\chi)$, and hence the relative projective element of $A$ with respect to $s$ is

$$
z_{A}=\sum_{\chi \in \operatorname{Irr}_{K}(A)} \sigma_{\chi}^{-1} \cdot \chi(1) \cdot e(\chi) .
$$

Suppose that (ii) holds; that is, we may assume that $s$ satisfies

$$
s=\pi^{-n} \rho=\sum_{\chi \in \operatorname{Irr}_{K}(A)} \pi^{-n} \cdot \chi(1) \cdot \chi .
$$

In that case, a trivial calculation shows that the associated relative projective element is, by the previous formula, equal to $\pi^{n} \cdot 1_{A}$. Thus (ii) implies (i).

Suppose finally that (iii) holds. We need to show that (ii) holds. For $U$ an $A$-lattice, write as before $K U=K \otimes_{\mathcal{O}} U$, and denote by $a(U)$ the smallest non-negative integer such that $\pi^{a(U)}$ annihilates $\underline{\operatorname{End}}_{A}(U)$. By the assumptions in (iii) and by Proposition 2.2 there is a non-negative integer $n$ such that

$$
\pi^{n} \cdot \operatorname{tr}_{K U}\left(z_{A}^{-1} \cdot \operatorname{End}_{A}(U)\right)=\operatorname{tr}_{U}\left(\operatorname{End}_{A}(U)\right)=\pi^{n-a(U)} \mathcal{O}
$$

for any $A$-lattice $U$. We apply this first to $U=A$. Since $A$ is projective as a left $A$-module, we have $a(A)=0$, and hence

$$
\pi^{n} \cdot \operatorname{tr}_{K A}\left(z_{A}^{-1} \cdot \operatorname{End}_{A}(A)\right)=\operatorname{tr}_{A}\left(\operatorname{End}_{A}(A)\right)=\pi^{n} \mathcal{O}
$$

Any $A$-endomorphism is given by right multiplication with an element $a$ in $A$. By elementary linear algebra, the trace of this endomorphism is equal to the trace of the linear endomorphism given by left multiplication with $a$, and hence this trace is equal to $\rho(a)$. $\operatorname{Thus~} \operatorname{tr}_{A}\left(\operatorname{End}_{A}(A)\right)=$ $\rho(A)=\pi^{n} \mathcal{O}$, which implies that $\pi^{-n} \rho$ sends $A$ to $\mathcal{O}$. Thus we have

$$
\pi^{-n} \rho=s_{w}
$$


for some $w \in Z(A)$. In order to show that $\pi^{-n} \rho$ is a symmetrising form on $A$ we need to show that $w \in Z(A)^{\times}$. Writing $w=\sum_{\chi \in \operatorname{Irr}_{K}(A)} \omega_{\chi} e(\chi)$ with coefficients $\omega_{\chi} \in \mathcal{O}$, we need to show that $\omega_{\chi} \in \mathcal{O}^{\times}$. A trivial calculation shows that

$$
s_{w}=\sum_{\chi \in \operatorname{Irr}_{K}(A)} \sigma_{\chi} \omega_{\chi} \chi
$$

Comparing coefficients with $\pi^{-n} \rho$ yields therefore

$$
\sigma_{\chi} \omega_{\chi}=\pi^{-n} \chi(1)
$$

for all $\chi \in \operatorname{Irr}_{K}(A)$, and hence

$$
\nu\left(\sigma_{\chi} \omega_{\chi}\right)=\nu\left(\pi^{-n} \chi(1)\right) .
$$

Let $\chi \in \operatorname{Irr}_{K}(A)$, and let $V$ be an $A$-lattice such that $K V=K \otimes_{\mathcal{O}} V$ has character $\chi$. Using that $\operatorname{End}_{A}(V)=\mathcal{O} \cdot \operatorname{Id}_{V}$, we get from the above that

$$
\nu\left(\pi^{n} \cdot \operatorname{tr}_{K V}\left(z_{A}^{-1}\right)\right)=\nu\left(\operatorname{Id}_{V}\right)=\nu(\chi(1)) .
$$

By the above formula for $z_{A}$, we have $z_{A}^{-1}=\sum_{\chi \in \operatorname{Irr}_{K}(A)} \sigma_{\chi} \cdot \chi(1)^{-1} \cdot e(\chi)$, and hence $\operatorname{tr}_{K V}\left(z_{A}^{-1}\right)=$ $\sigma_{\chi}$. Thus

$$
\nu\left(\pi^{n} \sigma_{\chi}\right)=\nu(\chi(1))
$$

Combining the previous statements yields

$$
\nu\left(\sigma_{\chi} \omega_{\chi}\right)=\nu\left(\pi^{-n} \chi(1)\right)=\nu\left(\sigma_{\chi}\right)
$$

and hence $\omega_{\chi}$ is invertible in $\mathcal{O}$. This shows that (iii) implies (ii). The last statement in Theorem 1.7 on the integer $n$ is obvious from the proofs of the implications.

Remark 3.1. The coefficients $\sigma_{\chi}^{-1}$ in the above proof are called Schur elements in [8, §7.2].

Next, we prove Theorem 1.8 As in the theorem, let $\operatorname{Irr}_{k}(A)$ denote an indexing set for the isomorphism classes of simple $k \otimes_{\mathcal{O}} A$-modules, and for $\chi \in \operatorname{Irr}_{K}(A)$ and $\varphi \in \operatorname{Irr}_{k}(A)$ denote by $d_{\chi, \varphi}$ the multiplicity of $S$ as a composition factor of $k \otimes_{\mathcal{O}} V$, where $V$ is an $A$-lattice such that $K \otimes_{\mathcal{O}} V$ has character $\chi$, and $S$ is a simple $k \otimes_{\mathcal{O}} A$-module with character $\varphi$. We adopt the analogous notation for other orders.

Lemma 3.2. Let $A^{\prime}$ be an $\mathcal{O}$-order which is derived equivalent to $A$. Then $\left|\operatorname{Irr}_{k}(A)\right|=\left|\operatorname{Irr}_{k}\left(A^{\prime}\right)\right|$, and $\left|\operatorname{Irr}_{K}(A)\right|=\left|\operatorname{Irr}_{K}\left(A^{\prime}\right)\right|$. Further, there exists a bijection $\chi \rightarrow \chi^{\prime}$ from $\operatorname{Irr}_{K}(A)$ to $\operatorname{Irr}_{K}\left(A^{\prime}\right)$, signs $\epsilon_{\chi} \in\{ \pm 1\}, \chi \in \operatorname{Irr}_{K}(A)$, and integers $u_{\varphi, \psi}, \varphi \in \operatorname{Irr}_{k}(A), \psi \in \operatorname{Irr}_{k}\left(A^{\prime}\right)$ such that

(i) For $\chi \in \operatorname{Irr}_{K}(A), \psi \in \operatorname{Irr}_{k}\left(A^{\prime}\right), d_{\chi^{\prime}, \psi}=\epsilon_{\chi} \sum_{\varphi \in \operatorname{Irr}_{k}(A)} d_{\chi, \varphi} u_{\varphi, \psi}$.

(ii) The form $s=\sum_{\chi \in \operatorname{Irr}_{K}(A)} \sigma_{\chi} \chi, \sigma_{\chi} \in K$ is a symmetrising form of $A$ if and only if the form $s^{\prime}=\sum_{\chi \in \operatorname{Irr}_{K}(A)} \epsilon_{\chi} \sigma_{\chi} \chi^{\prime}$ is a symmetrising form of $A^{\prime}$.

If $A$ and $A^{\prime}$ are Morita equivalent, then in addition there is a bijection $\varphi \rightarrow \varphi^{\prime}$ from $\operatorname{Irr}_{k}(A)$ to $\operatorname{Irr}_{k}\left(A^{\prime}\right)$ such that $d_{\chi^{\prime}, \varphi^{\prime}}=d_{\chi, \varphi}$ and $\epsilon_{\chi}=1$ for all $\chi \in \operatorname{Irr}_{K}(A), \varphi \in \operatorname{Irr}_{k}(A)$.

Proof. The first statement follows from [20, Theorem 6.8.8]. The transfer of symmetrising forms as in (ii) is proved in [7, Theorem 4.7]. 
Proof of Theorem 1.8. Suppose that the $\mathcal{O}$-order $A^{\prime}$ is Morita equivalent to $A$ and let $\chi \rightarrow \chi^{\prime}$, and $\varphi \rightarrow \varphi^{\prime}$ be the bijection of Lemma 3.2. Denoting by $n_{\varphi}$ the $k$-dimension of the simple $A^{\prime}$-module labelled by $\varphi^{\prime}\left(\varphi \in \operatorname{Irr}_{k}(A)\right)$, we have that $\chi^{\prime}(1)=\sum_{\varphi \in \operatorname{Irr}_{k}(A)} n_{\varphi} \cdot d_{\chi, \varphi}$ for all $\chi \in \operatorname{Irr}_{K}(A)$. The equivalence between (i) and (iii) is now immediate from Lemma 3.2 and the equivalence between (i) and (ii) of Theorem 1.7 We now prove that (iv) implies (iii). Let $n$ and $m_{\varphi}, \varphi \in \operatorname{Irr}_{k}(A)$ be integers such that $\pi^{-n} \sum_{\chi \in \operatorname{Irr}_{K}(A)} a_{\chi} \chi$ is a symmetrising form of $A$, where $a_{\chi}=\sum_{\varphi \in \operatorname{Irr}_{k}(A)} m_{\varphi} d_{\chi, \varphi}$, $\chi \in \operatorname{Irr}_{K}(A)$. Let $X$ be an $\mathcal{O}$-basis of $A$. Choose a positive integer $t$ such that $\pi^{-n} \cdot p^{t} \cdot d_{\chi, \varphi} \chi(x) \in \pi \mathcal{O}$ and $m_{\varphi}^{\prime}:=m_{\varphi}+p^{t}>0$ for all $\chi \in \operatorname{Irr}_{K}(A), \varphi \in \operatorname{Irr}_{k}(A)$ and $x \in X$. Set $s^{\prime}=\pi^{-n} \sum_{\chi \in \operatorname{Irr}_{K}(A)} a_{\chi}^{\prime} \cdot \chi$, where $a_{\chi}^{\prime}=\sum_{\varphi \in \operatorname{Irr}_{k}(A)} m_{\varphi}^{\prime} \cdot d_{\chi, \varphi}, \chi \in \operatorname{Irr}_{K}(A)$. Then for all $a \in A, s^{\prime}(a)-s(a) \in \pi \mathcal{O}$. Hence by considering the determinant of the Gram matrices of the bilinear forms associated to $s$ and $s^{\prime}$, it follows that $s^{\prime}$ is also a symmetrising form of $A$. This proves that (iii) holds. Since (i) clearly implies (ii) and (iii) implies (iv), in order to complete the proof, it suffices to show that (ii) implies (iv). Suppose that $A^{\prime}$ has the scalar projective property and that $A^{\prime}$ and $A$ are derived equivalent. Then (iii) holds for $A^{\prime}$, say for the integers $m_{\psi}, \psi \in \operatorname{Irr}_{k}\left(A^{\prime}\right)$. Then by Lemma 3.2, we have that (iv) holds for $A$ with the integers $n_{\varphi}=\sum_{\psi \in \operatorname{Irr}_{k}\left(A^{\prime}\right)} m_{\psi} u_{\varphi, \psi}, \varphi \in \operatorname{Irr}_{k}(A)$.

For the rest of this section we will expand on the question of the extent to which the characterisations of the projective scalar property up to Morita equivalence given in Theorem 1.8 (iii) and (iv) are constructive. The point here is that the set of symmetrising forms for an order $A$ is actually a $Z(A)^{\times}$-orbit, and $Z(A)$ is an $\mathcal{O}$-order for a (potentially) quite large ring $\mathcal{O}$. But in fact, as we will see, the criterion can be reduced to linear algebra over $\mathbb{Q}$.

The following proposition shows that the projective scalar property is essentially independent of the choice of the ring $\mathcal{O}$. This is particularly interesting to note since we often make the assumption that $K$ is a splitting field.

Proposition 3.3. Let $A$ be an $\mathcal{O}$-order and let $\mathcal{E} \supseteq \mathcal{O}$ be a discrete valuation ring containing $\mathcal{O}$ such that $J(\mathcal{E}) \cap \mathcal{O}=J(\mathcal{O})$. Then an $\mathcal{O}$-order $A$ has the projective scalar property if and only if the $\mathcal{E}$-order $\mathcal{E} \otimes_{\mathcal{O}} A$ has the projective scalar property.

Proof. By the characterisation in Theorem[1.7 $A$ having the projective scalar property is equivalent to some multiple of the regular trace being a symmetrising form for $A$. But the regular trace on $A$ and the regular trace of $\mathcal{E} \otimes_{\mathcal{O}} A$ have the same Gram-matrix (when the same basis is chosen for both of them), and invertibility of a multiple of said Gram-matrix over $\mathcal{O}$ is equivalent to invertibility over $\mathcal{E}$, provided of course that we multiplied by an element of $\mathcal{O}$.

So the only thing that still requires proof is that if $\tau$ is a generator of $J(\mathcal{E})$, then the integer $m$ such that $\tau^{-m} \cdot \rho$ is a symmetrising form for $\mathcal{E} \otimes_{\mathcal{O}} A$ satisfies $\tau^{m} \mathcal{E}=\pi^{n} \mathcal{E}$ for some $n \in \mathbb{Z}_{\geq 0}$ (since this means that $\pi^{-n} \cdot \rho$ is a symmetrising form for $A$ ). But by Theorem 1.7 we have $\tau^{m} \mathcal{E}=$ $\operatorname{tr}_{\mathcal{E} \otimes_{\mathcal{O}} A}\left(\operatorname{End}_{\mathcal{E} \otimes_{\mathcal{O}} A}\left(\mathcal{E} \otimes_{\mathcal{O}} A\right)\right)=\mathcal{E} \otimes_{\mathcal{O}} \operatorname{tr}_{A}\left(\operatorname{End}_{A}(A)\right)$, and $\operatorname{tr}_{A}\left(\operatorname{End}_{A}(A)\right)$ is certainly of the form $\pi^{n} \mathcal{O}$ for some $n$.

Definition 3.4. Let $A$ be an $\mathcal{O}$-algebra which is free of finite rank as an $\mathcal{O}$-module such that $K A$ is split semisimple. Fix an isomorphism

$$
\varphi: Z(K A) \stackrel{\sim}{\longrightarrow} K \times \ldots \times K
$$

We define the rational centre $Z^{\text {rat }}(K A)$ of $K A$ to be the $\mathbb{Q}$-algebra

$$
\varphi^{-1}(\mathbb{Q} \times \ldots \times \mathbb{Q})
$$


We define the rational centre of $A$, denoted by $Z^{\text {rat }}(A)$, as the intersection of $A$ with $Z^{\text {rat }}(K A)$.

We say that $A$ is rationally symmetric if there is an element

$$
\tilde{\sigma}=\sum_{\chi \in \operatorname{Irr}_{K}(A)} \tilde{\sigma}_{\chi} e_{\chi} \in Z^{\mathrm{rat}}(A)
$$

and an $n \in \mathbb{Z}$ such that

$$
\pi^{-n} \cdot \sum_{\chi \in \operatorname{Irr}_{K}(A)} \tilde{\sigma}_{\chi} \cdot \chi
$$

is a symmetrising form for $A$.

We should note that $\sigma_{\chi}=\pi^{-n} \cdot \tilde{\sigma}_{\chi}$ with $\sigma_{\chi}$ defined as earlier. Therefore rational symmetry is not the same as asking that the $\sigma_{\chi}$ be rational. Not even the projective scalar property implies rationality of the $\sigma_{\chi}$.

The rational centre of $A$ is a $\mathbb{Z}_{(p)}$-order, and the projective scalar property implies rational symmetry. We should remark that, if $\mathcal{O}$ is ramified over $\mathbb{Z}_{p}$, then rational symmetry is not necessarily preserved under direct sums. Neither is the projective scalar property, or even the property of being Morita-equivalent to an order which satisfies the projective scalar property. This is due to the possibility that the rational symmetrising forms involve different powers of $\pi$, whose quotient may have a non-integral $p$-valuation (using the convention $\nu(p)=1$ ).

Remark 3.5. An element $\tilde{\sigma}$ (together with an $n \in \mathbb{Z}$ ) as above and the central projective element $z_{A}$ are related by the formula

$$
z_{A}=\pi^{n} \cdot \tilde{\sigma}^{-1} \cdot \sum_{\chi \in \operatorname{Irr}_{K}(A)} \chi(1) \cdot e_{\chi}
$$

In particular, $\tilde{\sigma}$ can be chosen in $Z^{\text {rat }}(A)$ if and only if $z_{A} \in K^{\times} \cdot Z^{\text {rat }}(A)$. Now we can reinterpret the projective scalar property and rational symmetry in the following way: we consider the orbit $Z(A)^{\times} \cdot z_{A}$. If it intersects non-trivially with $K^{\times} \cdot Z^{\text {rat }}(A)$, then $A$ is rationally symmetric, and if it intersects non-trivially with $K^{\times} \cdot 1_{A}$, then $A$ has the projective scalar property.

In view of everything we have seen so far, the following is fairly straight-forward.

Proposition 3.6. Assume that $A$ is rationally symmetric, and $\tilde{\sigma} \in Z^{\mathrm{rat}}(A)$ is as before. Then $A$ has the scalar projective property if and only if

$$
\left\langle\sum_{\chi \in \operatorname{Irr}_{K}(A)} d_{\chi, \varphi} \cdot \chi \mid \varphi \in \operatorname{Irr}_{k}(A)\right\rangle \cap\left\{\sum_{\mathbb{Q} \in \operatorname{Irr}_{K}(A)} \tilde{\sigma}_{\chi} \cdot \frac{\chi(z)}{\chi(1)} \cdot \chi \mid z \in Z^{\mathrm{rat}}(A)\right\}
$$

properly contains

$$
\left\langle\sum_{\chi \in \operatorname{Irr}_{K}(A)} d_{\chi, \varphi} \cdot \chi \mid \varphi \in \operatorname{Irr}_{k}(A)\right\rangle \cap\left\{\sum_{\chi \in} \tilde{\sigma}_{\chi} \cdot \frac{\chi(z)}{\chi(1)} \cdot \chi \mid z \in I\right\}
$$

for all maximal Ideals $I$ in $Z^{\text {rat }}(A)$. 
Note that the right hand side in both (11) and (2) is the intersection of a $\mathbb{Q}$-vector space and a $\mathbb{Z}_{(p)}$-lattice, which can be computed by means of linear algebra.

We conclude this section with an example of a symmetric algebra which is not rationally symmetric, to show that the two notions are not equivalent.

Example 3.7. Assume that $k$ has characteristic two and $\mathcal{O}$ is unramified, i. e. $\pi=p=2$. Let $x \in \mathcal{O}^{\times}$be an arbitrary unit in $\mathcal{O}$. We consider the order $A=\left\langle\lambda_{1}, \lambda_{2}, \lambda_{3}, \lambda_{4}\right\rangle_{\mathcal{O}}$ in the commutative split-semisimple $K$-algebra $K \times K \times K \times K$, where

$$
\begin{aligned}
& \lambda_{1}=\left(\begin{array}{lllll}
1 & 1 & 1 & 1 &
\end{array}\right) \\
& \lambda_{2}=\left(\begin{array}{lllll}
0 & 2 & 0 & 2 x &
\end{array}\right) \\
& \lambda_{3}=\left(\begin{array}{lllll}
0 & 0 & 2 & 2 x &
\end{array}\right) \\
& \lambda_{4}=\left(\begin{array}{llll}
0 & 0 & 4 x
\end{array}\right)
\end{aligned}
$$

We claim that the map

$$
s: K \times K \times K \times K \longrightarrow K:\left(a_{1}, a_{2}, a_{3}, a_{4}\right) \mapsto \frac{2-x^{-1}}{4} a_{1}+\frac{1}{4} a_{2}+\frac{1}{4} a_{3}+\frac{x^{-1}}{4} a_{4}
$$

defines a symmetrising form for $A$. The Gram-matrix of $s$ with respect to the basis $\left(\lambda_{1}, \ldots, \lambda_{4}\right)$ is

$$
\left(s\left(\lambda_{i} \cdot \lambda_{j}\right)\right)_{i, j}=\left(\begin{array}{cccc}
1 & 1 & 1 & 1 \\
1 & 1+x & x & 2 x \\
1 & x & 1+x & 2 x \\
1 & 2 x & 2 x & 4 x
\end{array}\right)
$$

The determinant of this matrix is congruent to $1 \bmod 2 \mathcal{O}$, which implies that it is invertible over $\mathcal{O}$, which in turn implies that $A$ is a self-dual lattice with respect to $s$. So clearly, $A$ is a symmetric $\mathcal{O}$-order. However, if $x+2 \mathcal{O} \neq 1+2 \mathcal{O}$, then $A$ is not rationally symmetric. To see this we consider the family of forms

$$
s_{u}: K \times K \times K \times K \longrightarrow K:\left(a_{1}, a_{2}, a_{3}, a_{4}\right) \mapsto \frac{1}{4} \cdot \sum_{i=1}^{4} u_{i} \cdot a_{i}
$$

where $u \in\left(K^{\times}\right)^{4}$. By definition, the order $A$ is rationally symmetric if and only if $s_{u}$ is a symmetrising form for $A$ for some $u \in\left(\mathbb{Q}^{\times}\right)^{4}$. We know that the symmetrising forms for $A$ are exactly the forms $s(z \cdot-)$ with $z \in Z(A)^{\times}$and $s$ as in (44). The form $s(z \cdot-)$ is equal to $s_{z \cdot v}$ with $v=\left(2-x^{-1}, 1,1, x^{-1}\right)$. Since $z$ is a unit each $z_{i}$ lies in $\mathcal{O}^{\times}$, and so do all $v_{i}$. So if $A$ is symmetric with respect to $s_{u}$, then each $u_{i}$ needs to lie in $\mathcal{O}^{\times}$. Moreover, $A$ being symmetric with respect to $s_{u}$ would necessitate $A$ being integral with respect to $s_{u}$, which in particular would require $s_{u}\left(\lambda_{2}\right)=2^{-1} \cdot u_{2}+2^{-1} \cdot u_{4} \cdot x \in \mathcal{O}$. That is, $-\frac{u_{2}}{u_{4}}+2 \mathcal{O}=x+2 \mathcal{O}$, which can only hold true for rational $u_{i}$ 's if $x+2 \mathcal{O}$ lies in the prime field of $k$, which means $x+2 \mathcal{O}=1+2 \mathcal{O}$ (since we asked that $x$ be a unit, the case $x+2 \mathcal{O}=0+2 \mathcal{O}$ is impossible).

\section{Heights And Degrees of KnÖrR Lattices}

Proof of Proposition 1.9. Let $U$ be a Knörr lattice. Then, $\operatorname{tr}_{U}\left(\operatorname{End}_{A}(U)\right)=\operatorname{rank}(U) \mathcal{O}$. By Theorem 1.7 (iii), we have that $\operatorname{tr}_{U}\left(\operatorname{End}_{A}(U)\right)=\pi^{n-a(U)} \mathcal{O}$. By Theorem 1.7 (ii), we have

$$
\frac{\operatorname{rank}(A)}{\pi^{n}}=\frac{\rho\left(1_{A}\right)}{\pi^{n}} \in \mathcal{O} .
$$


It then follows that

$$
\operatorname{rank}(A) \mathcal{O} \subseteq \pi^{n} \mathcal{O} \subseteq \pi^{n-a(U)} \mathcal{O}=\operatorname{rank}(U) \mathcal{O} .
$$

This proves (i). Now suppose that $U$ is a projective lattice. Then, by Theorem 1.7 (iii) we have that $\operatorname{tr}_{U}\left(\operatorname{End}_{A}(U)\right)=\pi^{n} \mathcal{O}$. On the other hand, $\operatorname{rank}(U) \mathcal{O} \subseteq \operatorname{tr}_{U}\left(\operatorname{End}_{A}(U)\right)$. This proves (ii).

The next lemma is needed to prove Theorem 1.11.

Lemma 4.1. Let $A$ be a symmetric $\mathcal{O}$-algebra such that $K \otimes_{\mathcal{O}} A$ is split semisimple. Assume that $A$ has the projective scalar property and let $\pi^{n} 1_{A}$ be a relative projective element with respect to some symmetrizing form on $A$. Let $a_{0}=\max _{V}\{a(V)\}$ as $V$ ranges over all A-lattices. There exists $\chi \in \operatorname{Irr}_{K}(A)$ such that $\chi(1) \mathcal{O}=\pi^{n-a_{0}} \mathcal{O}$.

Proof. By Theorem 1.7(ii), $a_{0} \leq n$ and we have that $\chi(1) \mathcal{O} \subseteq \pi^{n-a_{0}} \mathcal{O}$ for all $\chi \in \operatorname{Irr}_{K}(A)$. Let $U$ be an $A$-lattice and $\alpha \in \operatorname{End}_{A}(U)$ be such that $\operatorname{tr}_{U}(\alpha) \mathcal{O}=\pi^{n-a_{0}} \mathcal{O}$. Let $f \in \mathcal{O}[x]$ be the characteristic polynomial of $\alpha$ and let $g \in \mathcal{O}[x]$ be an irreducible monic factor of $f$. Let $\bar{K}$ be an algebraic closure of $K$, let $\lambda_{i}, i \in \mathcal{I}$ be the roots of $g$ in $\bar{K}$ and for $i \in \mathcal{I}$ let $W_{i}$ be the generalised $\lambda_{i}$-eigen space of $\alpha$ in $\bar{K} \otimes_{\mathcal{O}} U$. Set $\lambda_{g}:=\sum_{i \in \mathcal{I}} \lambda_{i} \in \mathcal{O}$ and $W_{g}:=\oplus_{i \in \mathcal{I}} W_{i}$. We have $\operatorname{dim}\left(W_{i}\right)=\operatorname{dim}\left(W_{j}\right)=: d_{g}$ for all $i, j \in \mathcal{I}$. So,

$$
\operatorname{tr}_{W_{g}}(\alpha)=\lambda_{g} d_{g}
$$

Since $\operatorname{tr}_{U}(\alpha)$ is the sum of $\operatorname{tr}_{W_{g}}(\alpha)$ as $g$ runs through the irreducible factors of $f$ and since $\lambda_{g} \in \mathcal{O}$, replacing $g$ by some other irreducible factor of $f$ if necessary, we may assume that

$$
\pi^{n-a_{0}} \mathcal{O}=\operatorname{tr}_{U}(\alpha) \mathcal{O} \subseteq d_{g} \mathcal{O}
$$

Now $\alpha \in \operatorname{End}_{A}(U)$, hence $U_{i}$ is a $\bar{K} \otimes_{\mathcal{O}} A$-submodule of $\bar{K} \otimes_{\mathcal{O}} A$. In particular, $d_{g}$ is the dimension of a $\bar{K} \otimes_{\mathcal{O}} A$-module. Since $K \otimes_{\mathcal{O}} A$ is split it follows that there exists some $\chi \in I$ such that

$$
\pi^{n-a_{0}} \mathcal{O}=\operatorname{tr}_{U}(\alpha) \mathcal{O} \subseteq d_{g} \mathcal{O} \subseteq \chi(1) \mathcal{O} \subseteq \pi^{n-a_{0}} \mathcal{O} .
$$

Hence, $\chi(1) \mathcal{O}=\pi^{n-a_{0}} \mathcal{O}$ as desired.

Proof of Theorem 1.11. The fact that $U^{\prime}$ is a Knörr $A^{\prime}$-lattice is a consequence of Theorem 1.2. Let $a_{0}=\max _{V}\{a(V)\}$ as $V$ ranges over all $A$-lattices. Then $a_{0}$ also equals $\max _{V^{\prime}}\left\{a\left(V^{\prime}\right)\right\}$ as $V^{\prime}$ ranges over all $A^{\prime}$-lattices. Further, $a(U)=a\left(U^{\prime}\right)$. Let $\pi^{e} \mathcal{O}=p \mathcal{O}$ and let $\pi^{n} 1_{A}$ be a relative projective element of $A$. For any $A$-lattice $V, \operatorname{rank}(V) \mathcal{O} \subseteq \operatorname{tr}_{V}\left(\operatorname{End}_{A}(V)\right)$, hence by Lemma 4.1 and Theorem 1.7

$$
p^{\frac{n-a_{0}}{e}}=\min _{V}\left\{\operatorname{rank}(V)_{p}\right\}
$$

as $V$ ranges over all $A$-lattices. Since $U$ is a Knörr $A$-lattice and using again Theorem 1.7, it follows that

$$
\pi^{n-a(U)} \mathcal{O}=p^{\frac{n-a_{0}}{e}+h(U)} \mathcal{O}
$$

and hence

$$
h(U)=\frac{a_{0}-a(U)}{e} .
$$

Applying the same argument to $A^{\prime}$ and $U^{\prime}$ gives the desired result. 
Proof of Proposition 1.12. Let $u$ be an element of $U \backslash \pi U$. Let $\varphi: U \rightarrow U$ be an $\mathcal{O}$-linear projection onto $\mathcal{O} u$, and let $\operatorname{Tr}_{1}^{A}(\varphi)$ be the corresponding $A$-endomorphism of $U$. A calculation similar to that in Proposition 2.1 and using the assumption that $\pi^{n} 1_{A}$ is a relative projective element as in Theorem 1.7, shows that

$$
\operatorname{tr}_{U}\left(\operatorname{Tr}_{1}^{A}(\varphi)\right)=\pi^{n}
$$

Now because $U$ is projective, we have $a(U)=0$. It follows that

$$
\operatorname{tr}_{U}\left(\operatorname{End}_{A}(U)\right)=\pi^{n} \mathcal{O} .
$$

Because $U$ is a Knörr lattice, we can conclude that $\operatorname{Tr}_{1}^{A}(\varphi)$ is an invertible element of $\operatorname{End}_{A}(U)$. In particular, it is surjective. However, the image of $\operatorname{Tr}_{1}^{A}(\varphi)$ is contained in the $A$-lattice $A u$. We thus have $A u=U$. The result follows because $u$ was an arbitrary element of $U \backslash \pi U$.

\section{EXAMPLES}

Example 5.1. If $A=\operatorname{Mat}_{n}(\mathcal{O})$ for some positive integer $n$ or if $A=\mathcal{O} G$ for some finite group $G$, then $A$ has the scalar projective property (see [4, Examples and Remarks after Proposition 3.3]). If an $\mathcal{O}$-algebra $A$ has the projective scalar property, and if $B$ is a direct factor of $A$, then $B$ has the projective scalar property. This is immediate from the fact that the relative projective element with respect to a symmetrising form on $A$ is independent of the choice of an $\mathcal{O}$-basis. If $\mathcal{O}$-algebras $A$ and $B$ have the projective scalar property, then so does $A \otimes_{\mathcal{O}} B$. However, the projective scalar property is not preserved under taking direct products, whilst the property of being symmetric is. For instance if $p=2$, then by Proposition [1.9, $\mathcal{O} \times \operatorname{Mat}_{2}(\mathcal{O})$ does not have the projective scalar property. Further, $\mathcal{O} \times \operatorname{Mat}_{2}(\mathcal{O})$ is Morita equivalent to $\mathcal{O} \times \mathcal{O}$ from which we see that the scalar projective property is not invariant under Morita equivalence.

Example 5.2. Source algebras of blocks of finite groups have the projective scalar property. More precisely, if $A$ is a source algebra of a block of a finite group algebra with defect group $P$, and $k$ is a splitting field for the underlying finite group and its subgroups, then there is a symmetrising form on $A$ such that the relative projective element of $A$ is equal to $|P| \cdot 1$. To see this, let $G$ be a finite group, $B$ a block algebra of $\mathcal{O} G, P$ a defect group of $B$, and $i$ a source idempotent of $B$; that is, $i$ is a primitive idempotent in $B^{P}$ satisfying $\operatorname{Br}_{P}(i) \neq 0$, where $\operatorname{Br}_{P}:(\mathcal{O} G)^{P} \rightarrow k C_{G}(P)$ is the Brauer homomorphism. Assume that $k$ is a splitting field for $G$ and all of its subgroups. The source algebra $A=i \mathcal{O} G i$ is again symmetric, and any symmetrising form on $\mathcal{O} G$ restricts to a symmetrising form on $A$. Denote by $s: \mathcal{O} G \rightarrow \mathcal{O}$ the canonical symmetrising form, sending $1_{G}$ to $1_{\mathcal{O}}$ and $x \in G \backslash\left\{1_{G}\right\}$ to zero. With respect to this form, the relative trace $\operatorname{Tr}_{1}^{\mathcal{O} G}$ on $\mathcal{O} G$ is equal to the relative trace map $\operatorname{Tr}_{1}^{G}$, sending $a \in \mathcal{O} G$ to $\sum_{x \in G} x a x^{-1}$. The relative trace map $\operatorname{Tr}_{1}^{A}$ with respect to the symmetrising form $s$ restricted to $A$ satisfies $\operatorname{Tr}_{1}^{A}(a)=\operatorname{Tr}_{1}^{G}(a) i$. In particular, we have $\operatorname{Tr}_{1}^{A}(i)=\operatorname{Tr}_{1}^{G}(i) i$. As a consequence of [17] or [19, 9.3], the element $u=\operatorname{Tr}_{P}^{G}(i)$ is invertible in $Z(B)$. Moreover, we have $\operatorname{Tr}_{1}^{G}(i)=|P| \operatorname{Tr}_{P}^{G}(i)=|P| u$. Denote by $t$ the symmetrising form given by $t(a)=s(u a)$. The relative trace map on $A$ with respect to the form $t$ sends the unit element $i$ of $A$ to $|P| u u^{-1} i=|P| i$ as required.

Example 5.3. If $A$ is a Hopf algebra over $\mathcal{O}$ such that $K \otimes_{\mathcal{O}} A$ is semisimple, then $A$ has the projective scalar property. This is well-known to Hopf algebra experts-we just sketch the trail of ideas. By [11, Theorem 3.3] and [12, Theorem 4], the antipode of $K \otimes_{\mathcal{O}} A$ and of $K \otimes_{\mathcal{O}} A^{*}=$ $\left(K \otimes_{\mathcal{O}} A\right)^{*}$ has order 2. Hence the same is true for the antipode of $A$ and $A^{*}$. By the main 
theorem of [13, $A$ has a non-singular left integral say $\lambda$. Then $\lambda$ is also a non singular left integral for $K \otimes A$. Hence by [13, Props. 3 and 4] $\epsilon(\lambda) \neq 0$ and $A$ is unimodular. Since the antipode of $A^{*}$ also has order 2, by the second corollary to Proposition 8 of [13, applied with the roles of $A$ and $A^{*}$ reversed, we have that if $\Lambda \in A^{*}$ is a non-singular integral ( $\Lambda$ exists by the main theorem of [13] applied to $A^{*}$ ), then $\Lambda$ is a symmetrising form on $A$. Further, by [15, Section 5.3], the corresponding projective element is a scalar.

Example 5.4. This example shows that very few local commutative symmetric $\mathcal{O}$-algebras of $\mathcal{O}$-rank 2 have the projective scalar property. Let $A$ be an indecomposable $\mathcal{O}$-algebra such that $K \otimes_{\mathcal{O}} A=K \times K$; in particular, $A$ is commutative. Then there is a unique positive integer $m$ such that $A=\left\{(\alpha, \beta) \in \mathcal{O} \times \mathcal{O} \mid \beta-\alpha \in \pi^{m} \mathcal{O}\right\}=\left\{(\alpha, \alpha+\beta) \mid \alpha \in \mathcal{O}, \beta \in \pi^{m} \mathcal{O}\right\}$. The algebra $A$ is local commutative and symmetric, with symmetrising form $s$ sending $(\alpha, \alpha+\beta) \in A$ to $\pi^{-m} \beta$. We are going to show that $A$ has the projective scalar property if and only if $p=2$ and $2 \in \pi^{m} \mathcal{O}$.

The $\mathcal{O}$-basis $X=\left\{(1,1),\left(0, \pi^{m}\right)\right\}$ of $A$ has, with respect to $s$, the dual basis $\left\{\left(-\pi^{m}, 0\right),(1,1)\right\}$. Thus the relative projective element with respect to the symmetrising form $s$ is $z_{A}=\left(-\pi^{m}, \pi^{m}\right)$. We have $A^{\times}=\left\{\left(\alpha, \alpha+\pi^{m} \gamma\right) \mid \alpha \in \mathcal{O}^{\times}, \gamma \in \mathcal{O}\right\}$. Thus the $A^{\times}$-orbit of $z_{A}$ is $\left\{-\pi^{m} \alpha, \pi^{m} \alpha+\right.$ $\left.\pi^{2 m} \gamma \mid \alpha \in \mathcal{O}^{\times}, \gamma \in \mathcal{O}\right\}$. An element in this set is a scalar if and only if $\pi^{m} \gamma=-2 \alpha$. For $p$ odd this is impossible as the right side is invertible in $\mathcal{O}$ whereas the left side has a positive valuation of at least $m$. This shows that for $p$ odd, $A$ does not have the projective scalar property. For $p=2$, the algebra $A$ has the scalar property if and only if $\pi^{m}$ divides 2 in $\mathcal{O}$.

Note that since $A$ is local, any $\mathcal{O}$-algebra Morita equivalent to $A$ is a matrix algebra over $A$. Hence if $A$ does not have the projective scalar property, then neither does any algebra Morita equivalent to $A$.

Example 5.5. Let $(W, S)$ be a finite Coxeter group with length function $\ell$ and $q \in \mathcal{O}^{\times}$. Let $\mathcal{H}=\mathcal{H}_{q}(W, S)$ be the associated Iwahori-Hecke algebra over $\mathcal{O}$ with parameter $q$. That is, $\mathcal{H}$ has an $\mathcal{O}$-basis $\left\{T_{w}\right\}_{w \in W}$, with multiplication given by $T_{w} T_{y}=T_{w y}$ if $w, y \in W$ such that $\ell(w y)=$ $\ell(w)+\ell(y)$, and $\left(T_{s}\right)^{2}=q T_{1}+(1-q) T_{s}$ for $s \in S$. By [8, Proposition 8.1.1], the algebra $\mathcal{H}$ is symmetric, with a symmetrising form sending $T_{1}$ to 1 and $T_{w}$ to 0 for $w \in W \backslash\{1\}$. The dual basis of $\left\{T_{w}\right\}_{w \in W}$ with respect to this form is $\left\{q^{-\ell(w)} T_{w^{-1}}\right\}_{w \in W}$, and hence the associated relative projective element is

$$
z_{\mathcal{H}}=\sum_{w \in W} q^{-\ell(w)} T_{w} T_{w^{-1}}
$$

Whether $\mathcal{H}$ has the projective scalar property seems to be difficult to read off this expression. If $p=2$ and $W=S_{2}=S=\{1, s\}$, and if $q$ is an odd integer, then the map sending $T_{1}$ to $(1,0)$ and $T_{s}$ to $(1,1-q)$ is an injective algebra homomorphism from $\mathcal{H}$ to $\mathcal{O} \times \mathcal{O}$. The previous example shows that $\mathcal{H}$ has the scalar property if and only if $q \equiv 3 \bmod 4$.

Example 5.6. Let $G$ be a finite group and assume that $\mathcal{O}$ contains the values of all irreducible characters of $G$. Let $A=\mathcal{O}[\operatorname{Irr}(G)]=\mathcal{O} \otimes_{\mathbb{Z}} \mathbb{Z}[\operatorname{Irr}(G)]$. The irreducible characters of $G$ form an $\mathcal{O}$-basis for $A$. For $K$-valued functions $\alpha$ and $\beta$ on $G$, define the usual

$$
[\alpha, \beta]=\frac{1}{|G|} \sum_{g \in G} \alpha(g) \beta\left(g^{-1}\right) \in K .
$$

For $\chi \in \operatorname{Irr}(G)$, let $\bar{\chi}$ denote the character of the contragredient representation, so $\bar{\chi}(g)=\chi\left(g^{-1}\right)$ for all $g \in G$. Finally, let $1_{G}$ denote the trivial character of $G$. 
For $\chi, \psi \in \operatorname{Irr}(G)$, the identity $\left[\chi \bar{\psi}, 1_{G}\right]=[\chi, \psi]=\delta_{\chi, \psi}$ implies that the $\mathcal{O}$-linear function $s: A \rightarrow \mathcal{O}$ given by $s(\alpha)=$ (coefficient of $1_{G}$ in $\alpha$ ) is a symmetrizing form on $A$. The same identity makes it clear that the basis of $A$ dual to $\operatorname{Irr}(G)$ with respect to $s$ is given by $\chi^{\vee}=\bar{\chi}$. The corresponding relative projective element, $z=\sum_{\chi \in \operatorname{Irr}(G)} \chi \bar{\chi}$, coincides with the function on $G$ sending $g$ to $\left|C_{G}(g)\right|$. Clearly, $z$ is a scalar multiple of $1_{G}$ if and only if $G$ is abelian. In this case, we have $z=|G| \cdot 1_{G}$. However, to see exactly when $A$ has the projective scalar property, it is necessary to consider the action of $A^{\times}$on $z$. Let $u$ be an invertible element of $A$. Then $u$ is a function from $G$ to $\mathcal{O}^{\times}$. Assume that $u z=\lambda \cdot 1_{G}$ for some element $\lambda \in \mathcal{O}$. We must then have

$$
u(g)=\frac{\lambda}{\left|C_{G}(g)\right|} \in \mathcal{O}^{\times}
$$

for all $g \in G$. Thus, $\left|C_{G}(g)\right|_{p}$ is independent of $g$. We deduce that every element of $G$ must centralise a Sylow $p$-subgroup. So, let $P$ be a Sylow $p$-subgroup. By Sylow's theorem, every element of $G$ is conjugate to an element of $C_{G}(P)$. A well known application of "Burnside's counting lemma" allows us to conclude that $C_{G}(P)=G$. Thus, $P$ is abelian, and $G \cong P \times H$ for some group $H$ of $p^{\prime}$ order. Conversely, we claim that if $G=P \times H$, with $P$ an abelian $p$-group and $H$ a group of $p^{\prime}$-order, then $A$ has the projective scalar property. All that remains to do is to verify that the function $u(g)=\frac{1}{\left|C_{G}(g)\right|}$ for $g \in G$ actually lies in $A$, assuming $G=P \times H$ as above. So let $\chi \in \operatorname{Irr}(G)$. We must show that $[\chi, u] \in \mathcal{O}$. We can write $\chi=\theta \otimes \psi$ for irreducible characters $\theta$ of $P$ and $\psi$ of $H$. One verifies

$$
[\chi, u]=\left\{\begin{array}{cl}
\frac{1}{|H|} \sum_{h \in H} \frac{\psi(h)}{\left|C_{H}(h)\right|} & \text { if } \theta=1_{P} \\
0 & \text { if } \theta \neq 1_{P}
\end{array}\right.
$$

In both cases, we have $[\chi, u] \in \mathcal{O}$.

Finally, we remark that if $\mathcal{O}$ is a Dedekind domain in which no prime dividing the order of $G$ is invertible, then $A$ has the scalar projective property if and only if $G$ is abelian.

Example 5.7. The Knörr property is not preserved by Morita equivalences in general. The idea is that all absolutely indecomposable $A$-lattices of $p^{\prime}$-rank are Knörr, but among those of rank divisible by $p$, only the absolutely irreducible lattices tend to have the property. Indeed, the proof of [9. Corollary 1.6] does not require the $\mathcal{O}$-algebra to be a group ring (nor even a symmetric algebra). Thus, any Morita equivalence that sends a lattice of $p^{\prime}$-rank which is indecomposable but not irreducible to a lattice of rank divisible by $p$ is likely to give an example.

Specifically, let $p=2$ and assume that $\mathcal{O}$ is unramified and $k$ is algebraically closed. Let $A$ be the principal block algebra of $\mathcal{O} A_{5}$, where $A_{5}$ is the alternating group of degree 5 . Then $\left|\operatorname{Irr}_{K}(A)\right|=4$, $\left|\operatorname{Irr}_{k}(A)\right|=3$, and the decomposition matrix of $A$ with respect to some ordering of $\operatorname{Irr}_{K}(A)$ is

\begin{tabular}{cccc} 
& $\varphi_{1}$ & $\varphi_{2}$ & $\varphi_{3}$ \\
\hline$\chi_{1}$ & 1 & 0 & 0 \\
$\chi_{2}$ & 1 & 0 & 1 \\
$\chi_{3}$ & 1 & 1 & 0 \\
$\chi_{4}$ & 1 & 1 & 1
\end{tabular}

where $\varphi_{1}$ corresponds to a 1 -dimensional $k A$-module, and $\varphi_{2}$ and $\varphi_{3}$ correspond to simple $k A$ modules of dimension 2.

For each $i, 1 \leq i \leq 3$, let $P_{i}$ denote a projective indecomposable $A$-module such that $P_{i} / \operatorname{rad}\left(P_{i}\right)$ is isomorphic to a simple $k A$-module corresponding to $\varphi_{i}$. Let $e$ be an idempotent in $A$ such that 
$A e \cong P_{1}+2 P_{2}+P_{3}$ as left $A$-modules. Then $A$ and $e A e$ are Morita equivalent via the functor sending an $A$-module $M$ to the $e A e$-module $e M$ and an $A$-module $\alpha: M \rightarrow N$ homomorphism to the $e A e$-module homomorphism $e \cdot \alpha: e M \rightarrow e N$ defined through restriction to $e M$. The simple $e A e$-modules corresponding to $\varphi_{1}$ and $\varphi_{3}$ have dimension 1 whereas the simple $e A e$-module corresponding to $\varphi_{2}$ has dimension 2 .

From the decomposition matrix above, one sees that the character afforded by $K P_{1}$ has two irreducible constituents, one of degree 5 and the other of degree 3. It follows from [9, Lemma 1.9] that $P_{1}$ is not a Knörr $A$-lattice. However, the rank of the $e A$-lattice $e P_{1}$ is 7 . Thus $e P_{1}$ is a Knörr eAe-lattice.

To obtain an example in which neither lattice is projective, it is enough to inflate the $P_{i}$ above to lattices for the group $A_{5} \times C_{2}$, where $C_{2}$ is a cyclic group of order 2 .

Notice also that although $e P_{1}$ is Knörr, it does not have the stable exponent property. This is the case for both the $A_{5}$ and $A_{5} \times C_{2}$ situations. Next, we produce a lattice with the stable exponent property which is not Knörr.

First, we have $\mathbb{Q}(\sqrt{5}) \subseteq K$, so $K A$ is split semisimple. Let $M$ be the unique quotient lattice of $P_{1}$ such that $K M$ has character $\chi_{1}+\chi_{2}+\chi_{3}$. Since $M$ has rank 7, $M$ is a Knörr $A$-lattice. Because $A$ has the projective scalar property, $M$ and hence $e M$ also have the stable exponent property. We shall show that $e M$ is not Knörr.

Let $L$ be the unique $\mathcal{O}$-free quotient of $M$ affording the character $\chi_{3}$ and let $\alpha: M \rightarrow L$ be the projection map. Since $\alpha$ is surjective, and $L$ and $M$ are not projective, $\alpha \notin \operatorname{Hom}_{A}^{\mathrm{pr}}(M, L)$. Thus, by Corollary 1.4, there exists $\beta \in \operatorname{Hom}_{A}(L, M)$ such that $\operatorname{tr}_{M}(\beta \circ \alpha) \notin 4 \mathcal{O}$ (since $4 \cdot 1_{A}$ is a projective scalar element of $A$ ).

Let $\tau=\beta \alpha$ and denote also by $\tau$ the $K$-linear extension of $\tau$ to $K M$. For each $i, 1 \leq i \leq 4$, let $e_{i}$ be the primitive central idempotent of $K A$ corresponding to $\chi_{i}$. Since $\tau(K M)$ is contained in $e_{3}(K M)$, we have that $\left(e_{2}+e_{4}\right)(K M)$ is contained in the kernel of $\tau$. On the other hand, $1-e=\left(e_{2}+e_{4}\right)(1-e)$. Thus, $\operatorname{tr}_{K M}(\tau)=\operatorname{tr}_{e(K M)}(\tau)$. It follows that

$$
\operatorname{tr}_{e M}(e \cdot \tau)=\operatorname{tr}_{K M}(\tau)=\operatorname{tr}_{M}(\tau) \notin 4 \mathcal{O}
$$

Since $e M$ has rank 6 , we have that $\nu_{2}\left(\operatorname{tr}_{e M}(e \cdot \tau)\right) \leq \nu_{2}\left(\operatorname{rank}_{\mathcal{O}}(e M)\right)$. Since $\tau$ is not invertible, neither is $e \cdot \tau$, hence $e M$ is not a Knörr $e A e$-lattice.

Example 5.8. Let $\mathcal{O}=\mathbb{Z}_{3}$, and consider the $\mathcal{O}$-order $A=\mathcal{O} S_{3}$, that is, the group ring of the symmetric group on three points. The decomposition matrix of $A$ is

\begin{tabular}{ccc} 
& $\varphi_{(3)}$ & $\varphi_{(2,1)}$ \\
\hline$\chi_{(3)}$ & 1 & 0 \\
$\chi_{(2,1)}$ & 1 & 1 \\
$\chi_{\left(1^{3}\right)}$ & 0 & 1
\end{tabular}

Here we use the standard indexing of ordinary and modular irreducible characters of symmetric groups via partitions. Let $e_{(3)}, e_{(2,1)}$ and $e_{\left(1^{3}\right)}$ denote the primitive idempotents in $Z(K A)$. The inertial index of this block is two, and, according to [1, that means that this block has six isomorphism types of indecomposable lattices (one can also show this in an elementary way). It is also easy to enumerate those isomorphism types: there are two indecomposable projective lattices, which are non-irreducible. Then there is a unique lattice with character $\chi_{(3)}$ and a unique lattice with character $\chi_{\left(1^{3}\right)}$. Moreover there is a lattice with character $\chi_{(2,1)}$ whose top has Brauer character $\varphi_{(3)}$ and there is a lattice with character $\chi_{(2,1)}$ whose top has Brauer character $\varphi_{(2,1)}$ (those 
two lattices are the projective lattices over the order $\left.A e_{(2,1)}\right)$. As there are but six lattices in total we know that there can be no further indecomposable lattices. In particular, all indecomposable lattices are either projective or absolutely irreducible. This implies that each algebra in the Morita equivalence class of $A$ has the property that Knörr-lattices and absolutely indecomposable nonprojective lattices with the stable endomorphism property coincide. Any algebra in the Morita equivalence class of $A$ which does not possess the projective scalar property will therefore provide a counterexample to the converse of Theorem 1.2 .

Choose $B$ in the Morita equivalence class of $A$ such that the Morita equivalence sends the simple module with character $\varphi_{(3)}$ to a one-dimensional module and the simple module with character $\varphi_{(2,1)}$ to a two-dimensional module. Note that

$$
\frac{1}{3} \cdot\left(\chi_{(3)}(-)+2 \cdot \chi_{(2,1)}(-)+\chi_{\left(1^{3}\right)}(-)\right)
$$

is a symmetrising form for $A$, and therefore also for $B$ (with the characters replaced by the corresponding characters of $B$ ). It follows that

$$
z_{B}=3 \cdot\left(e_{(3)}+\frac{3}{2} \cdot e_{(2,1)}+2 \cdot e_{\left(1^{3}\right)}\right)
$$

and this element is determined uniquely up to multiplications by units in $Z(A)=Z(B)$. But multiplication by units cannot turn the above element into a scalar, since it will leave the 3 valuation of the coefficients of the idempotents $e_{(3)}, e_{(2,1)}$ and $e_{\left(1^{3}\right)}$ invariant. Hence $B$ does not possess the projective scalar property.

\section{REFERENCES}

[1] C. Bessenrodt, Indecomposable lattices in blocks with cyclic defect groups. Comm. Algebra 10 (1982), no. 2, 135-170.

[2] M. Broué, Isométries parfaits, types de blocs, catégories dérivées. Astérisque No. 181-182 (1990), 61-92.

[3] M. Broué, Equivalences of Blocks of Group Algebras, in: Finite dimensional algebras and related topics, Kluwer (1994), 1-26.

[4] M. Broué, Higman's criterion revisited. Michigan Math. J. 58 (2009), 125-179.

[5] K. Brown, Cohomology of groups. Graduate texts in mathematics 87, Springer Verlag New York (1982).

[6] J. F. Carlson and A. Jones An exponential property of lattices over group rings. J. London Math. Soc. 39 (1988), 467-479.

[7] F. Eisele, p-Adic lifting problems and derived equivalences J. Algebra 356 (2012), 90-114.

[8] M. Geck and G. Pfeiffer, Characters of finite Coxeter groups and Iwahori-Hecke algebras. Oxford University Press, Oxford (2000).

[9] R. Knörr, Virtually irreducible lattices. Proc. London Math. Soc. 59 (1989), 99132.

[10] R. Knörr, Projective homomorphisms of RG-lattices, unpublished preprint.

[11] R.G. Larson and D.E. Radford Finite dimensional cosemisimple Hopf algebras in characteristic 0 are semisimple J. Algebra 117 (1988), 267-289.

[12] R.G. Larson and D.E. Radford Semisimple cosemisimple Hopf algebras Amer. J . Math. 110 (1988), 187-195.

[13] R.G. Larson and M.E. Sweedler, An associative orthogonal bilnear form for Hopf algebras. Amer. J. Math 91 (1969), 7594.

[14] A. Jacoby, M. Lorenz, Frobenius divisibility for Hopf Algebras, Contemporary Mathematics.

[15] M. Lorenz, Some applications of Frobenius algebras to Hopf algebras. Contemporary Mathematics 537, (2011) 269-289.

[16] H. Nagao and Y. Tsushima, Representations of finite groups, Academic Press (1989).

[17] C. Picaronny and L. Puig, Quelques remarques sur un thème de Knörr. J. Algebra 109 (1987) 69-73. 
ON TATE DUALITY AND A PROJECTIVE SCALAR PROPERTY FOR SYMMETRIC ALGEBRAS

[18] K. Roggenkamp, The construction of almost split sequences for integral group rings and orders. Comm. Algebra 5 (1977), 1363-1373.

[19] J. Thévenaz, Duality in G-algebras. Math. Z. 200 (1988), 4785.

[20] A. Zimmermann, Representation Theory: A homological algebra point of view. Algebra and Applications, Springer, Cham (2014)

City University London, Northampton Square, London, EC1V 0HB, UK

E-mail address: Florian.Eisele@city.ac.uk

Northern Illinois University, DeKalb, IL 60115, USA

E-mail address: mgeline@niu.edu

City University London, Northampton Square, London, EC1V 0HB, UK

E-mail address: Radha.Kessar.1@city.ac.uk

City University London, Northampton Square, London, EC1V 0HB, UK

E-mail address: Markus.Linckelmann.1@city.ac.uk 